\title{
Influence of Depression And Self-Esteem On Suicidal Ideation Among University Students
}

\author{
Ajayi Olubukola \\ Department of psychology \& behavioural studies \\ Faculty of the social sciences, ekiti state university, ado ekiti.
}

\begin{abstract}
The research examined the influence of self- esteem and depression on suicidal ideation among university students. The research was carried out among university students to measure the influence of self- esteem and depression on suicidal ideation. Two hundred and fifty one participants [251] were sampled from a university for the purpose of this research. The instrument used for this research was questionnaire. The statistical methods used were t-test and one way ANOVA. Four hypotheses were tested and the result revealed: Students with high selfesteem [M=12.7877] significantly reported lower suicidal ideation than those with low self- esteem [M =18.5905], df [249] = -6.043, $p<.01$. The result implies that self-esteem influenced suicidal ideation among students. The results also shows that students with low depression [M = 12.8188] significantly reported lower suicidal ideation than those with high depression $[M=19.5222]$, df $[248]=6.903, p<.01$. The result implies that depression influenced suicidal ideation among students. Likewise the result shows that female students [M=16.1301] were not significantly different from male students [M = 14.3359], df [249] $=1.780, p>.05$. The result implies that there is no gender difference in suicidal ideation among students. However students in 100 level of study [M =15.3469] were not significantly different in suicidal ideation compared to those in $200[\mathrm{M}=14.7375], 300[\mathrm{M}=14.5000], 400[\mathrm{M}=$ $15.8036]$ and $500[M=19.1000]$ levels of study. The result means that the level of study has no significant influence on suicidal ideation among students $F[4,246]=0.846 ; p>.05$.
\end{abstract}

Key words: Depression, Self-esteem Suicidal ideation.,

\section{INTRODUCTION}

Suicide is one of those unsolved psychosocial issue which cause a lot of pain to those who are left behind due to their dealing of guilt and grief about their behaviours with their loved ones (Parker \& Benson, 2004). The effective prediction of suicidal death remains to be a problematic issue, and it has been a taboo topic all over the world especially in conservative countries like Nigeria. After completing suicide, it may be impossible to know the internal thoughts or motives that drove any individual to commit suicide. Since suicidal ideation occur among variety of people (young and old, rich and poor, educated or less educated), it can be very difficult to pin point a typical suicidal ideation profile among university students. Such worldwide problem of suicidal ideation has been considered a significant factor leading to death according to many researchers (Yao, et al; 2008). 
Despite the ranking by the World Happiness report of Nigeria as the 91st out of the 156 countries, suicide and suicidal related behaviours have been on the increase. More worrisome is the fact that the case is alarming among university students. For instance, in the first lap of 2017, there were reported cases of suicide and suicidal attempts in both private and public universities in Nigeria (Aruya, 2017). Reason for such behaviour has become a main scientific concern to both behavioural researchers and health practitioners. Although, university life is characterized with several challenges that may prone students to many abnormal behavioural outcomes, suicidal ideation may not readily come to mind as an option to coping with such challenges.

Depression is a serious mental health problem that may lead to different ripple effects in students. One of such ripple effects is suicidal ideation or suicidal thoughts which may lead to suicide attempts or suicide. Suicidality or suicidal behaviour exists along a continuum that extends from suicidal ideation or thoughts, suicide related communications, suicide attempts and finally suicide (CDCP, 2008).

Depression is one of the most common and recurrent mood disorders, which presently affects 340 million individuals worldwide. The main signs include: low mood, feelings of worthlessness, guilt or inability to think or work productively (Schlebusch, Burrows and Wada, 2009). However, symptoms change with time and their intensity depends on patient's developmental stage. The etiopatogenesis of depression is not yet fully understood, but its onset is believed to depend on the influence of numerous risk factors, such as socio-economic, environmental, cultural, ethnic, poor academic performance, emotional trauma, loss of beloved one or genetic determinants. Depressive disorders are considered to be one of the most disabling conditions, which not only impair daily functioning of affected individuals and their families, but also create an enormous burden for society (Alesi, Rappo and Pepi, 2014).

Furthermore, studies have demonstrated that between $40-80 \%$ of students were clinically depressed at the time of their suicide attempts. Also, depression, especially in young people, was connected with susceptibility to alcohol addiction, drug abuse, crime, and a range of other adverse phenomena. Although depression beginning at an early age could have serious developmental and functional consequences, such as academic failure or persistent psychosocial problems, it remained misdiagnosed, undertreated, or even ignored (Andrew, 2010). Subsequently, examination of this phenomenon has its theoretical and practical implications because assessment of the risk of depression and its determinants is crucial for the preparation of accurate prevention and treatment programmes. Important life events or major changes are believed to make individuals particularly susceptible to depression or depressive symptoms. Here, some authors propose that students should be analyzed separately because of distress connected with study years (Bandura, et al, 1999). They also indicate that time spent in university marks a transition from adolescence to young adulthood, when individuals go through psychophysical processes typical for this stage of life. Additionally, they pass through psychosocial changes, such as leaving home or separation from one's family and friends and adjustment to a new system of learning.

Studies have reported that self-esteem is a powerful internal protective factor against students' suicide behaviours (Eskin et al., 2007). Fergusson et al. (2003) found that resiliency to suicidal behaviours was associated with increased self-esteem among young people. It is strongly believed that suicidal behaviours affect many among university students all over the world. Self- esteem 
Olubukola, A. (2020) Influence of Depression And Self-Esteem On Suicidal Ideation Among University Students. Advances in Social Sciences Research Iournal, 7(4) 318-325.

levels can be an important issue during the first year of college students' life. People with low self esteem develop a feeling to harm themselves because they are not living up to their expectations that may be developed internally by the students and externally by other (Kaur, \& Rani, (2012). People usually preoccupied with the thoughts of suicidal ideation to overcome and block unbearable emotional pain, caused by a wide variety of stressful life problems (Whitlock, \& Knox, 2007).

Self-esteem is the sum of attitudes which depend on perceptions, thoughts, evaluations, feelings and behavioural tendencies aimed toward ourselves, the way we are and behave, and our body's and character's features. The biggest influence on self-esteem is parenting, acceptance, and control. In other words, it's one self's evaluative perception. Young children have relatively high self-esteem, which gradually declines over the course of childhood. As children develop cognitively, they begin to base their self-evaluations on external feedback and social comparisons. As they grow older, they form a more balanced and accurate appraisal of their academic competence, social skills, attractiveness, and other personal characteristics. Self-esteem continues to decline during adolescence which has been attributed to the adolescent body image and other problems associated with puberty. Adolescence is the critical period for the development of self-esteem and self-identity, and low self-esteem may endanger adolescents' emotional regulation (Tsang, Yip, 2006).

\section{Research Participants}

\section{RESEARCH}

Participants that were used in this study comprised of University Students from Ekiti State University, Ado-Ekiti within Ekiti State. The sample of the present study comprised of 251 undergraduates selected from Ekiti State University, Ado-Ekiti (including male and female).A questionnaire was administered to each student who was willing to participate in the research. Random sampling technique was used in this study.

\section{Research Instruments}

The following three self-reported questionnaires were administered to all participants in the study.

\section{Suicidal Ideation Scale (SIS)}

The Suicidal Ideation Scale (SIS; Rudd, 1989) is a 10 item self-report measure designed to assess the severity or intensity of suicidal ideation among university students. Using a 5-point Likert scale ( $1=$ never or none of the time; $5=$ always or great many times) participants report the extent to which each statement is true of the way they have felt or behaved in the past year. The SIS was designed to represent a continuum of suicidal ideation ranging from covert suicidal thoughts ("Life is so bad I feel like giving up") to more overt or intense suicidal ideation ("I have been thinking of ways to kill myself"). All items are scored in a positive direction and the SIS yields a single score, with higher scores representing greater levels of suicidal ideation. Among a sample of 215 university students, the SIS demonstrated high levels of internal consistency reliability (coefficient alpha $=.90$ ), with item-total correlations ranging from .45 to .74 . The reliability alpha for internal consistency on the SIS was 90 . 


\section{The Rosenberg Self-Esteem Scale}

The Rosenberg Self-Esteem (RSE) Scale was developed by Rosenberg (1965). This scale consisted of self-worth statements ranging from low self-esteem to high self-esteem. The scale is a ten item Likert type. Items are four point scale ranging from strongly agree to strongly disagree. Strongly agree was given four points, agree three points, disagree two points, and strongly disagree one point. Scoring: The scale consisted of five positive items and five negatives items. Negative items (2, $5,6,8$, and 9) were reversed scored. The score of the students ranged IUGJEPS 23(2) (2015) a1-a15 Omar Al-Shawashereh from (0 to 40). The possible score divided into three levels, low level from (0 - 1.33), moderate level from (1.34 - 2.67), and high level from (2.68-4.0). The reliability of the scale established through the overall measure of the internal consistency which was obtained by (Cronbach alpha $=0.78$ ). The high score on the scale meant a higher level of self-esteem. This scale is well known measure of self- esteem all over the world. Rosenberg (1965) reported internal consistency reliability (Cronbach alpha) ranging from 0.85 to 0.88 for the participating students.

\section{The Beck Depression Inventory}

The Beck Depression Inventory (Beck, Steer \& Brown, 1996): The Beck Depression Inventory is a widely utilized 21-item self-report scale in both clinical and research studies. The Beck Depression Inventory-II is a depression rating scale that can be used in individuals that are ages 13 years and older, and rates symptoms of depression in terms of severity on a scale from 0 to 3 based on the 21 specific items. The test-retest reliability for BDI-II was 0.93 .

\section{PROCEDURE FOR DATA COLLECTION}

The questionnaires were administered to the respondents by the researcher personally, the participants were from the Faculty of Law, Faculty of Education, Faculty of Social sciences, Faculty of Art, Faculty of Science, Faculty of Engineering, Faculty of Management sciences, Faculty of Agriculture and College of Medicine cutting across different levels in the university. The researcher made sure that the undergraduates filled the questionnaire without discussing. The respondents were not be given any time to respond to the instruments. The completed questionnaires were retrieved from the participants immediately. The researcher's challenge during the research was that many of the undergraduates were not interested in the study but they were educated on the importance of the research.

\section{Statistical analysis}

The results were mainly on the data obtained from the questionnaires. Hypothesis one, two and three were tested using t-test while hypothesis four was tested using one-way ANOVA.

\section{RESULTS}

The collected data were properly scored and analyzed; the results were discussed based on the hypotheses generated in the study.

\section{Hypothesis One}

Hypothesis one stated that students with high self-esteem would significantly report lower suicidal ideation than those with low self-esteem. The hypothesis was tested using t-test for independent samples. The result is presented in Table 4.1. 
Olubukola, A. (2020) Influence of Depression And Self-Esteem On Suicidal Ideation Among University Students. Advances in Social Sciences Research Iournal, 7(4) 318-325.

Table 1: t-test Summary Table showing influence of self-esteem on suicidal ideation

\begin{tabular}{|c|c|c|c|c|c|c|c|}
\hline \multicolumn{2}{|c|}{ Self-esteem } & $N$ & Mean & $S D$ & $D f$ & $T$ & $\boldsymbol{P}$ \\
\hline Suicidal & High & 146 & 12.7877 & 4.4601 & 249 & -6.043 & $<.01$ \\
\hline ideation & Low & 105 & 18.5905 & 10.3484 & & & \\
\hline
\end{tabular}

From Table 1 , the result shows that students with high self-esteem $(M=12.7877)$ significantly reported lower suicidal ideation than those with low self-esteem $(M=18.5905), d f$ $(249)=-6.043, p<.01$. The result implies that self-esteem influenced suicidal ideation among students. Therefore, hypothesis one was confirmed in the study.

\section{Hypothesis Two}

Hypothesis two stated that students with low depression would significantly report lower suicidal ideation than those with high depression. The hypothesis was tested using t-test for independent samples. The result is presented in Table 2.

Table 2: t-test Summary Table showing influence of depression on suicidal ideation

\begin{tabular}{|c|c|c|c|c|c|c|c|}
\hline \multicolumn{2}{|c|}{ Depression } & $N$ & Mean & SD & Df & T & P \\
\hline \multirow{3}{*}{ Suicide Ideation } & High & 90 & 19.5222 & 10.8007 & 248 & 6.903 & $<.01$ \\
\cline { 2 - 8 } & Low & 160 & 12.8188 & 4.4082 & & & \\
\hline
\end{tabular}

From Table 2 , the result shows that students with low depression $(M=12.8188)$ significantly reported lower suicidal ideation than those with high depression $(M=19.5222), d f(248)=6.903, p$ $<.01$. The result implies that depression influenced suicidal ideation among students. Therefore, hypothesis two was confirmed in the study.

\section{Hypothesis Three}

Hypothesis three stated that male students would significantly report lower suicidal ideation than female students. The hypothesis was tested using t-test for independent samples. The result is presented in Table 3.

Table 3: t-test Summary Table showing influence of gender on suicidal ideation

\begin{tabular}{|c|c|c|c|c|c|c|c|}
\hline \multicolumn{2}{|c|}{ Gender } & $N$ & Mean & $S D$ & $D f$ & $T$ & $P$ \\
\hline \multirow{2}{*}{ Suicide Ideation } & Male & 123 & 16.1301 & 9.2780 & 249 & 1.780 & $>.05$ \\
\hline & Female & 128 & 14.3359 & 6.5053 & & & \\
\hline
\end{tabular}

From Table 3, the result shows that female students $(M=16.1301)$ were not significantly different in suicidal ideation compared to male students $(M=14.3359), d f(249)=1.780, \mathrm{p}>.05$. The result implies that there is no gender difference in suicidal ideation among students. Therefore, hypothesis three was not confirmed in the study. 


\section{Hypothesis Four}

Hypothesis four stated that students in 100 level would significantly report lower suicidal ideation than those in 200, 300, 400 and 500 levels. The hypothesis was tested using One-way Analysis of Variance. The result is presented in Table 4.4.

Table 4: Summary of One-Way ANOVA Table showing influence of level of study on suicidal ideation

\begin{tabular}{|c|c|c|c|c|c|}
\hline Source & Sum of Square & df & Mean Square & F & Sig \\
\hline Between Groups & 218.054 & 4 & 54.513 & 0.846 & $>.05$ \\
\hline Within Groups & 15860.329 & 246 & 64.473 & & \\
\hline Total & 16078.383 & 250 & & & \\
\hline
\end{tabular}

Results in Table .4 show that students in 100 level of study $(M=15.3469)$ were not significantly different in suicidal ideation compared to those in $200(M=14.7375), 300(M=14.5000), 400(M=$ $15.8036)$ and $500(M=19.1000)$ levels of study. It means that level of study has no significant influence on suicidal ideation among students $F(4,246)=0.846$; $p>05)$. Therefore, hypothesis four was not confirmed.

\section{DISCUSSION}

The study focused on the influence of depression, self-esteem on suicidal ideation among university students. The study aimed to find out the relationship between depression and suicidal ideation among university students, to find out the relationship between students' suicidal ideation and their level of self-esteem, to identify the factors that lead to depression which in turn leads to suicidal ideation among university students and to find out the implication of sex on self-esteem, depression and suicidal ideation.

The study assumed four hypotheses on the influence of depression, self-esteem on suicidal ideation among university students. Hypothesis one stated that students with high self-esteem would significantly report lower suicidal ideation than those with lower self-esteem. The result implies that self-esteem influenced suicidal ideation among students. This result is in accordance with the findings of Dukes and Lorch, (1989) who opined that low self-esteem has been found to be important in the prediction of suicidal ideation among students. Brent et al, (1986) also added that the presence and severity of suicidal ideation in students has been related to low self-esteem. Yao et al. (2014) who showed that higher self-esteem was significantly associated with suicidal ideation. Hypothesis two stated that students with low depression would significantly report lower suicidal ideation than those with high depression. The result implies that depression influenced suicidal ideation among students. This finding is similar to Katz, Chapman and Chun, (2010) who found that depression is higher in those who have school problems. World Health Statistics, (2016) added that depression is the cause of suicidal ideation among young adults.

The third Hypothesis stated that male students would significantly report lower suicidal ideation than female students. The result implies that there is no gender difference in suicidal ideation among students. These findings is similar to Grossman and Kruesi, (2000) who found that suicidal 
Olubukola, A. (2020) Influence of Depression And Self-Esteem On Suicidal Ideation Among University Students. Advances in Social Sciences Research Iournal, 7(4) 318-325.

ideation is a common phenomenon among all individuals irrespective of age, gender, ethnicity, race, academic performance or even social status.

The last hypothesis stated that students in 100 level would significantly report lower suicidal ideation than those in 200, 300, 400 and 500 level. It means that level of study has no significant influence on suicidal ideation among students. The outcome is as result of the fact that students are generally exposed to different forms of pressure such as academic pressure, family pressure and also peer pressure from the study observation.

\section{CONCLUSION}

In conclusion, suicide is a complicated phenomenon made up of several intricacies of cognitive processes. The act of suicide is never a sudden event. Several factors, events and contemplations occur before an attempt could be considered. Some of these factors may be psychological, biological, psychosocial or an interaction of all the factors. However, in the continuum of the interaction of such factors or cognitive processes, suicidal thoughts or ideation are most times the first steps leading to eventual suicide.

Suicidal ideation is a definite pointer to imminent suicide among individuals experiencing such. It is a psychological reaction to interactions with the social environment hence influenced by social interactions with the cognition of an individual. Hence, the psychosocial factors in this study were considered as influencers of the cognition of students that may be experiencing suicidal ideation. The findings from this study depict self-esteem as the most influential psychosocial factor. Selfesteem is a value system an individual has of ones' self, a value system based on a sense of selfworth by the individual. The lower this level of self-worth the higher the intensity of suicidal ideation as seen from statistical analyzed in this study. Self-esteem may also influence the individuals' optimism to always have positive expectations about life. Depression being a major symptom of suicidal behaviour is an outcome of psychosocial interactions where the individual has a sense of self-worthlessness and is pessimistic about outcomes of life's events.

This study provides a basis for further investigations to take place in order to elucidate several factors that may be influencing suicidal ideation among students and adolescents alike. This would go a long way to preventing individuals taking their own lives and enhance mental health, giving life a meaning to those who have lost sight of it.

\section{RECOMMENDATIONS AND LIMITATIONS}

The department for student support system should consider it essential to launch early psychological interventions to improve most especially optimism and sense of self-worth among students as protective factors against suicidal ideation. Forums could be created and made open frequently for students experiencing feelings of giving up on life or any major risk factor especially depression to access help to improve mental health of students. The prevalence of suicidal ideation may be low and suicide even lower, but no case irrespective of its intensity should be over looked. Social networks could be used to reach personal level interventions, where individuals would be more comfortable to share their mental health concerns.

Considering the religious nature of the school, further studies could be tailored to assess the influence of religious activities on the level of suicidal ideation among students on the campus. The 
researcher's challenge during the research was that many of the undergraduates were not interested in the study.

\section{References}

Alesi, M., Rappo, G., \& Pepi, A. (2014). Depression, anxiety at School and self esteem in children with learning disabilities. Psychology of Abnormal Child 3: 125. Doi: 10.4172/2329-9525. 1000125.

Andrews, L.W. (2010). Encyclopaedia of depression Vol 1 (A-L). California: Greenwood Publishing Group.

Aruya, T. (2017). Nigeria and increasing rate of suicide. This Day Newspaper online; April, 21

Bandura, A., Pastorelli, C., Barbaranelli, C. \& Caprara, G.V. (1999). Self efficacy pathways $\quad$ to childhood depression. Journal of Personal Social Psychology, 76(2) $258-269$.

Beck, A.T, Steer, R.A. \& Brown, G.K (1996). Manual for the Beck Depression Inventory - 11. San Antonio, TX: Psychological Corporation.

Centre for Disease Control and Prevention (CDCP) (2008). Suicide facts at a glance. http://www. cdc.gov/ violence prevention /pdf/suicide-datasheet-.pdf.

Eskin, M., Ertekin,K.,Dereboy, C. \& Demikiran, F.(2007). Risk factors for and protective factors $\quad$ against adolescent suicidal behavior in Turkey. Crisis; The journal of crisis intervention and suicide prevention, 28(3), 131-139.

Fergusson DM, Horwood LJ, Swain-Campbell NR, (2003). Panics attacks and Psychoticism

Psychol. MED .Jan; 33(1): 15-21.

Grossman, J.A., \& Kruesi,M.J.P [2000]. Innovative approaches to youth suicide prevention: an update of issues and research findings. In R.W. Maris, S.S. Canetto, J.L. McIntosh,\& M.M. Silverman [eds.], suicidology, 2000 [P.170-201]. Guiford press.

Katz, E.R., Chapman, L.L., \& Chun, T.N. (2010). Psychiatric emergencies in Fleisher, G.R \& Ludwig, S (Eds). Textbook of Pediatric Emergency Medicine. Philadelphia, Lippincott Williams and Wilkins \& Wolters Kluwer.

Kaur, Mandeep \& Rani, Anju (2012). Suicidal ideation, self- esteem and Perfectionism: The vicious triad. Voice of Research, 1, (2), 52-57.

Parker, J.,\& Benson, M. (2004). Parent-adolescent relations and adolescent functioning: Self-esteem, substance abuse, and delinquency. Adolescence, 39(155), 519-530.

Rosenberg, M. (1965).Society and the adolescent self-image. Princeton, NJ: Princeton University Press. IUGJEPS 23(2) (2015) a1-a15 Omar Al-Shawashereh .

Schlebusch, L., Burrows, S. \& Wada, N. (2009). Suicide prevention and religious traditions on the African Continent. In D. Wasserman \& C. Wasserman (Eds) Suicidality and suicide prevention. A global perspective. Oxford, England. Oxford University Press.

SKM Tsang, \& FYY Yip. (2006).Development and validation of positive youth development scale. International journal of Adolescent Medicine and Health 18(3), 459-466.

World Health Organization (2016) world health statistics.(2016) Monitoring health for the SDGs, Sustainable development goals. World health organization.

Whitlock, J., \& Knox, K. (2007). The relationship between self-injurious behavior and suicide in a young adult population. Archive of Pediatric Adolescence Medical, 161(7), 634-640.

Yao,Y-S.,Chang,W-W., Jim, ,Y-L., Chen,Y.,He,L-P.\& Zang, L.(2014). Life satisfaction, Coping, Self-Esteem and Suicidal ideation in Chinese Adolescents: A school -based study. Child: Care, Health and Development, 40, 747-752. 